\title{
Urine exosomes biomarkers of Obesity treatment: A pilot study
}

Bhavya Vijay ( $\sim$ bhavya.v@tdu.edu.in )

The university of trans-disciplinary health sciences and technology

Satish P Ramachandra Rao

The university of trans-disciplinary health sciences and technology

Poornima Devkumar

The university of trans-disciplinary health sciences and technology

\section{Research Article}

Keywords:

Posted Date: January 31st, 2022

DOI: https://doi.org/10.21203/rs.3.rs-1290415/v1

License: (1) This work is licensed under a Creative Commons Attribution 4.0 International License.

Read Full License 


\section{Urine exosomes biomarkers of Obesity treatment: A pilot study}

Bhavya Vijay, Satish P Ramachandra Rao, Poornima Devkumar

${ }^{1}$ The University of Trans-Disciplinary Health Sciences and Technology, Bangalore, India.

Corresponding Author: Bhavya Vijay, bhavya.v@tdu.edu.in

\section{Abstract}

\section{Background}

Obesity is a raising risk factor for many diseases including Cardiovascular risks and Cancer. The present limitations and inaccuracy of anthropometric measures as well as treatment options demand research of integrative management strategies and identification of specific biomarkers of obesity. This study identifies the urine exosome biomarkers and its differential expression profile between 3-timepoints from female obese participants who underwent Ayurveda treatment.

\section{Methodology}

Urine exosomes were isolated using PEG methods of isolation. The major proteins obtained were identified using label-free quantification in LC-MS/MS. The genes involved in pathway enrichment, functional annotation and disease manifestation were identified and shortlisted based on protein interaction network. The hub genes representing obesity are identified.

\section{Results}

The exosomes isolated were validated using LC-MS/MS. Using UniProt as reference, a total of 210 exosome proteins were identified. 73 genes were overexpressed in pathway enrichment analysis. Further, GO functional annotation identified 15 common genes involved. Finally, the 8 genes common in obesity were identified and its differential expression profile was seen to have corrected at timepoints during treatment. 


\section{Conclusion}

This is the first study to isolate and identify urine exosomes profiles from female obese participants of India. The study results indicate the correction in the differential expression profile of 8 hub genes involved in obesity post treatment. The biomarker signature of the pilot study indicates the specificity of urine exosomes as diagnostic markers. The study also promotes the potential of integrative and complementary treatment approaches like Ayurveda in effective management of many metabolic diseases.

\section{Introduction}

World Health Organisation (WHO) defines Obesity in adults as BMI greater than or equal to $30 \mathrm{~kg} / \mathrm{m}^{2}$. The global prevalence of obesity has tripled since 1975 covering approximately $39 \%$ of the global adult population (1). Women are much prone to obesity compared to men. As per National Family Health Survey (NFHS)-3, India reports approximately 13\% of the women as obese between age 15-59 years (2). Raising risk of diabetes(3), hypertension(4), cardiovascular diseases(5) and cancer(6) due to obesity makes it a huge disease burden. Currently, the most common measure of obesity is BMI, which is a surrogate marker for obesity, as it measures only the body fatness(7). BMI values along with waist circumference values have become the standard obesity assessments in clinical practice(8). However, a number of studies have shown that in some populations, waist circumference shows only a relative risk of obesity due to ethnicity-specific confounders(9). Hence, the cut-off ranges of waist measurements in obesity have been stratified for various ethnic groups(10). Novel research methods are aiming at identifications of different biological indicators such as inflammatory markers, stress, genetic, nutritional and gut microbiota markers to identify effective treatments for obesity. Current markers in obesity treatment, study on early miRNA markers have shown a potential for characterizing and modifying obesity and diet patterns(11-13).

Another interesting and novel field of biomarkers are Exosomes. Exosome's specificity as biomarkers of health and disease is well documented, with some studies also focusing on exosome biomarkers of obesity(14). As exosome component of almost any kind of biofluid is a practical feasibility, it has potential utility for cell-specific and focussed prognosis of the targeted cells. Exosomes from adipose tissues were used to identify obesity markers using many studies. Expression profiling of the exosomes revealed miRNA markers of metabolic diseases such as cardiovascular illnesses, atherosclerosis, diabetes etc(15). However, most biomarker studies are pre-clinical and controlled, only limited number of proteomic marker 
studies have been conducted in a prospective clinical study to understand the mechanism of biomarker variation during the course of treatment.

To address this gap, this study focussed on identification of potential biomarker of obesity where the subjects had undergone an Ayurveda treatment (Lekhana Basti), an established Ayurveda regimen, in treating obesity using the 24-hour urine samples collected during the treatment for the exosome proteomic biomarker analysis.

\section{Materials and Methods}

\section{Chemicals and software tools:}

Chemicals: Phosphate buffer saline pH 7.4 (HiMedia, Cat no: TL1099), ECP buffer, Bovine Serum Albumin (BSA) (HiMedia, Cat no: MB083), Bradford's reagent (HiMedia, Cat no: ML106), Sodium Dodecyl Sulfate (Merck), Tris base (HiMedia, Cat no: MB029), Acrylamide (GeNei, Cat no: ER13), Bis-acrylamide (GeNei, Cat no: ER14), Ammonium persulphate (GeNei, Cat no: ER11B), TEMED, 2X Laemmli buffer (HiMedia, Cat no: ML021), acetic acid (Loba, Cat no: L0019502500), Commassie Brilliant Blue R-250 (HiMedia, MB153), Acetonitrile, ammonium bicarbonate, Trypsin, water (HPLC grade, Himedia, Cat no: AS028, GRM1021, RM618, AS077), pre-stained molecular marker (HiMedia, Cat no: MBT092), formic acid, beta-mercaptoethanol (Sigma Aldrich, Cat no: M6250), methanol (Sigma Aldrich, Cat no: 179337), pH 7, pH 4 and pH 10 calibrators (Eutech, Cat no: FCP62/4.0, 7.0, 10.0) were used.

Consumables and Equipment's: Micro-centrifuge tubes (Tarsons, Cat no: T500010), pipette tips (Tarsons, Cat no: T521020 \& T521010), 15ml and 50ml centrifuge tubes (Tarsons, Cat no: T546021 \& T546041), pH meter (Hanna Instruments, Cat no: HI98107), SDS plates and comb (BioRad, Cat no: 1653311, 1653308, 1653359), Gel run system (Mini-PROTEAN Tetra Cell, Biorad, Cat no: 1658004EDU), rocking shaker (Rocky max, Tarsons, Cat no: T4080), heating block (Riviera, Cat no: 50031001), Power pac (Bio-Rad PowerPac 300 electrophoresis power supply, Cat no: 68192-1), vortex shaker (Spinix, Tarsons, Cat no: T3020), mini centrifuge (Spinwin, Tarsons, Cat no: T1010) and Refrigerated high speed centrifuge (Eltek, Cat no: RC4100F) were used for isolation procedures.

Software and database tools: For the data analysis, MSConvert GUI platform, progenesis software tool, Mascot server, The KEGG, DAVID and Panther pathway analysis tools, STRING protein interaction network tool, DisGeNeT database, GO functional annotation database, Cytoscape and Tableau were used. 


\section{Sample collection and processing:}

A total of $n=18$ 24-hour secondary urine samples from 6 female participants were obtained considering the sample availability and data for analysis. The samples were obtained from the clinical study conducted at IAIM Healthcare, Bangalore on "Effect of Ayurveda based enema on intestinal permeability and endotoxin assay in obesity study" (unpublished work). The samples were centrifuged and stored at $-80^{\circ} \mathrm{C}$ before exosome isolation.

\section{Urine exosome protein isolation and gel run:}

The exosomes were isolated using standard Polyethylene Glycol based exosome isolation protocols $(16,17)$. Bradford's method of protein estimation was performed and $250 \mu \mathrm{g}$ protein. Using 12.5\% Acrylamide, SDS-PAGE was run and the trypsinised as per standard methods(18). The trypsinised mixtures were subjected for label-free protein quantification with LC-MS/MS unit.

\section{Mass Spectrometry analysis:}

LC-MS/MS protein quantification was done using Thermo Orbitrap nano LC-MS/MS unit. $5 \mu 1$ of the sample was injected into the inlet, with MS/MS ion search type, unrestricted protein mass input, 10ppm peptide mass tolerance, and 0.6Da fragment mass tolerance, a maximum of 2 missed cleavages were allowed, and a False Discovery Rate (FDR) of $<1 \%$ with significance threshold $\mathrm{p}<0.05$. Reference sequence was obtained from UniProt (UP000005640).

\section{Mass Spectrometry Data Proteomic analysis:}

The raw files were converted into readable/ open file formats (such as .mzML format) using MSConvert GUI software interface. All the data files were analysed for protein identification using the Progenesis QI software, from Waters, MA.

During the alignment and quality check, 2 subject files which did not match the minimum alignment were rejected. A total of 12 files, from 4 subjects were carried for further analysis. To remove irregularities, the $\mathrm{m} / \mathrm{z}$ reads only between $10-75$ minutes of the run were selectively filtered. As the samples are time-point samples from same subjects, the input samples were defined into three groups as Before, During and After treatment samples. Using MASCOT server, proteins were identified against reference UniProt Homo sapiens ID (UP00005640). To determine the correlations between groups, a repeated-measures ANOVA was performed to 
identify the normalized abundances of the peptides across the time-points. (See Supplementary Table-1 for full result)

\section{PPI Network}

A protein-protein interaction network was built using the online tool STRING, to determine the key proteins involved in Obesity at treatment time-points. The confidence score for the interactions was set to $>0.7$ to filter only the proteins with only strong interactions.

\section{Pathway analyses and functional annotation:}

KEGG pathway enrichment analysis was performed using STRING tool at FDR $<0.05$. Further, PANTHER tool was used to determine statistical overrepresentation patterns at FDR $<0.05$. Gene functional annotation analysis was performed using GO database to identify the key genes involved in the biological processes, molecular functions and cellular components. The common genes from the 3 functional groups, pathways and PPI network were filtered.

\section{Disease-gene relationship:}

To determine the genes directly linked with obesity condition, a list of genes associated with obesity was extracted from DisGeNet database. The common genes obtained fKEGG enrichment and GO functional annotations were compared with the obesity gene reference (C0028754 from DisGeNet) to determine the key genes interacting in Obesity. Finally, the genes involved in Obesity from literature were compared to our data and mapped for similar expression patterns.

\section{Results and Discussion}

All the participants of the study were female with an average age of $43.1 \pm 7.3$ years. The clinical outcome parameters (secondary data) indicated significant reduction in anthropometric indicators of obesity such as weight, BMI, waist and hip circumference (Figure 1) 


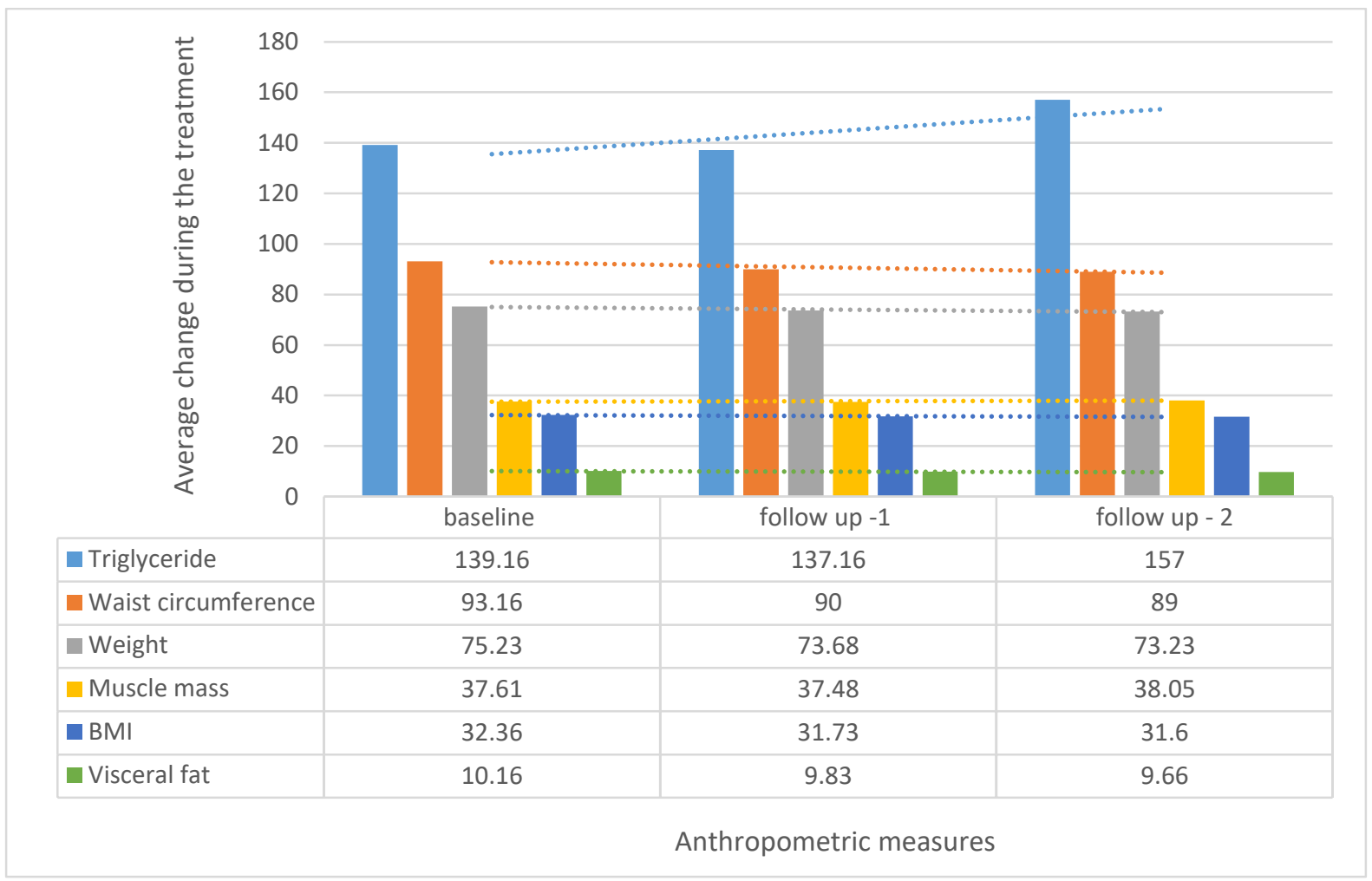

*Average age of the subjects $-43.1 \pm 7.3$ years

Figure 1: Demographics of the samples

After LC-MS/MS analysis, a final list of 210 proteins were seen in all the three sample groups and its respective normalised abundance scores were obtained. STRING protein-protein interaction network provided a strong interaction network at the confidence of $>0.7$ (See Figure $2 \& 3)$. 


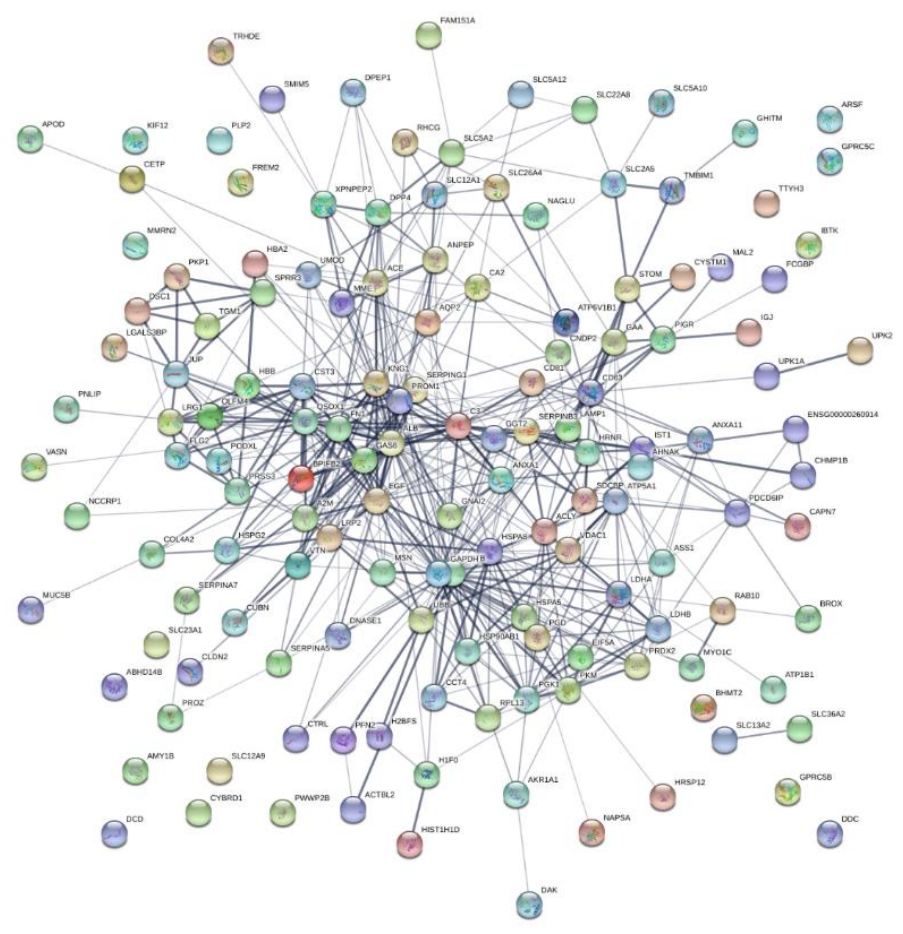

Figure 2: STRING protein-protein interaction network

The KEGG pathway enrichment analysis from the interacted proteins showed 12 pathways closely related to the proteins. Proteins from the KEGG analysis is highlighted in the Figure 3 of string interaction network. Further, from Panther over-representation analysis, 31 molecular pathways were over-represented as shown in Table 1.

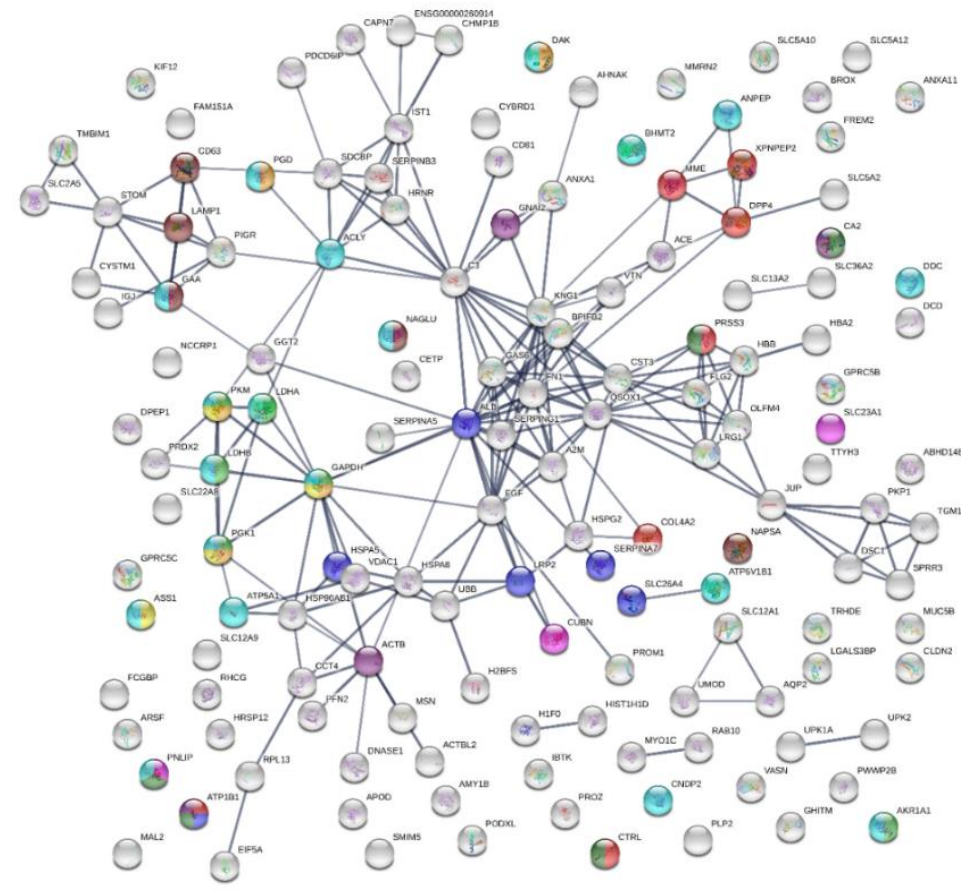

Figure 3: Protein from enriched pathways in STRING Network 
Table 1: Over-represented molecular pathways from PANTHER

\section{SI. GO molecular function complete FDR}

no.

\begin{tabular}{llll}
\hline $\mathbf{1}$ & metallodipeptidase activity (GO:0070573) & 0.04360 \\
\hline $\mathbf{2}$ & $\begin{array}{l}\text { dipeptidyl-peptidase activity (GO:0008239) } \\
\mathbf{3}\end{array}$ & $\begin{array}{l}\text { monosaccharide transmembrane transporter } \\
\text { activity (GO:0015145) }\end{array}$ & 0.003690 \\
\hline $\mathbf{4}$ & $\begin{array}{l}\text { sugar transmembrane transporter activity } \\
\text { (GO:0051119) }\end{array}$ & 0.00442 \\
\hline $\mathbf{5}$ & & \\
\hline
\end{tabular}

\begin{tabular}{lllll}
\hline 5 & $\begin{array}{l}\text { carbohydrate:cation } \\
\text { (GO:0005402) }\end{array}$ & symporter & activity & 0.04020 \\
\hline $\mathbf{6}$ & $\begin{array}{l}\text { hexose transmembrane } \\
\text { (GO:0015149) }\end{array}$ & transporter & activity & 0.04440 \\
\hline $\mathbf{7}$ & $\begin{array}{l}\text { glucose transmembrane } \\
\text { (GO:0005355) }\end{array}$ & transporter & activity & 0.04280 \\
\hline 8 & carbones & & \\
\hline
\end{tabular}

8 carbohydrate transmembrane transporter activity 0.00179 (GO:0015144)

\begin{tabular}{llll}
\hline $\mathbf{9}$ & aminopeptidase activity (GO:0004177) & 0.00044 \\
\hline $\mathbf{1 0}$ & $\begin{array}{l}\text { cysteine-type endopeptidase inhibitor activity } \\
\text { (GO:0004869) }\end{array}$ & 0.00666 \\
\hline $\mathbf{1 1}$ & metalloexopeptidase activity (GO:0008235) & 0.00792 \\
\hline $\mathbf{1 2}$ & solute:sodium symporter activity (GO:0015370) & 0.00232 \\
\hline $\mathbf{1 3}$ & exogenous protein binding (GO:0140272) & 0.01690 \\
\hline $\mathbf{1 4}$ & solute:cation symporter activity (GO:0015294) & 0.00167 \\
\hline $\mathbf{1 5}$ & exopeptidase activity (GO:0008238) & 0.00195 \\
\hline $\mathbf{1 6}$ & endopeptidase inhibitor activity (GO:0004866) & 0.00026 \\
\hline $\mathbf{1 7}$ & peptidase inhibitor activity (GO:0030414) & 0.00018 \\
\hline $\mathbf{1 8}$ & endopeptidase regulator activity (GO:0061135) & 0.00015 \\
\hline $\mathbf{1 9}$ & peptidase regulator activity (GO:0061134) & 0.00042 \\
\hline $\mathbf{2 0}$ & symporter activity (GO:0015293) & 0.00572 \\
\hline $\mathbf{2 1}$ & protease binding (GO:0002020) & 0.02330 \\
\hline $\mathbf{2 2}$ & sodium ion transmembrane transporter activity & 0.04440
\end{tabular}
(GO:0015081)

\begin{tabular}{lll}
$\mathbf{2 3}$ & cadherin binding (GO:0045296) & 0.00148 \\
\hline $\mathbf{2 4}$ & $\begin{array}{l}\text { secondary active transmembrane transporter } \\
\text { activity (GO:0015291) }\end{array}$ & 0.01490 \\
\hline $\mathbf{2 5}$ & $\begin{array}{l}\text { active ion transmembrane transporter activity } \\
\text { (GO:0022853) }\end{array}$ & 0.01750 \\
\hline $\mathbf{2 6}$ & cell adhesion molecule binding (GO:0050839) & 0.00024 \\
\hline $\mathbf{2 7}$ & enzyme inhibitor activity (GO:0004857) & 0.00370 \\
\hline $\mathbf{2 8}$ & structural molecule activity (GO:0005198) & 0.00320 \\
\hline
\end{tabular}




\begin{tabular}{|lll|}
\hline $\mathbf{2 9}$ & calcium ion binding (GO:0005509) & 0.03700 \\
\hline $\mathbf{3 0}$ & enzyme regulator activity (GO:0030234) & 0.04570 \\
\hline $\mathbf{3 1}$ & molecular_function (GO:0003674) & 0.00155 \\
\hline
\end{tabular}

From the GO functional annotation, a total of 25 genes were found common among Molecular, Biological and Cellular functions shown in Figure 4, $5 \& 6$. The final list of common genes from all the three functions are shortlisted

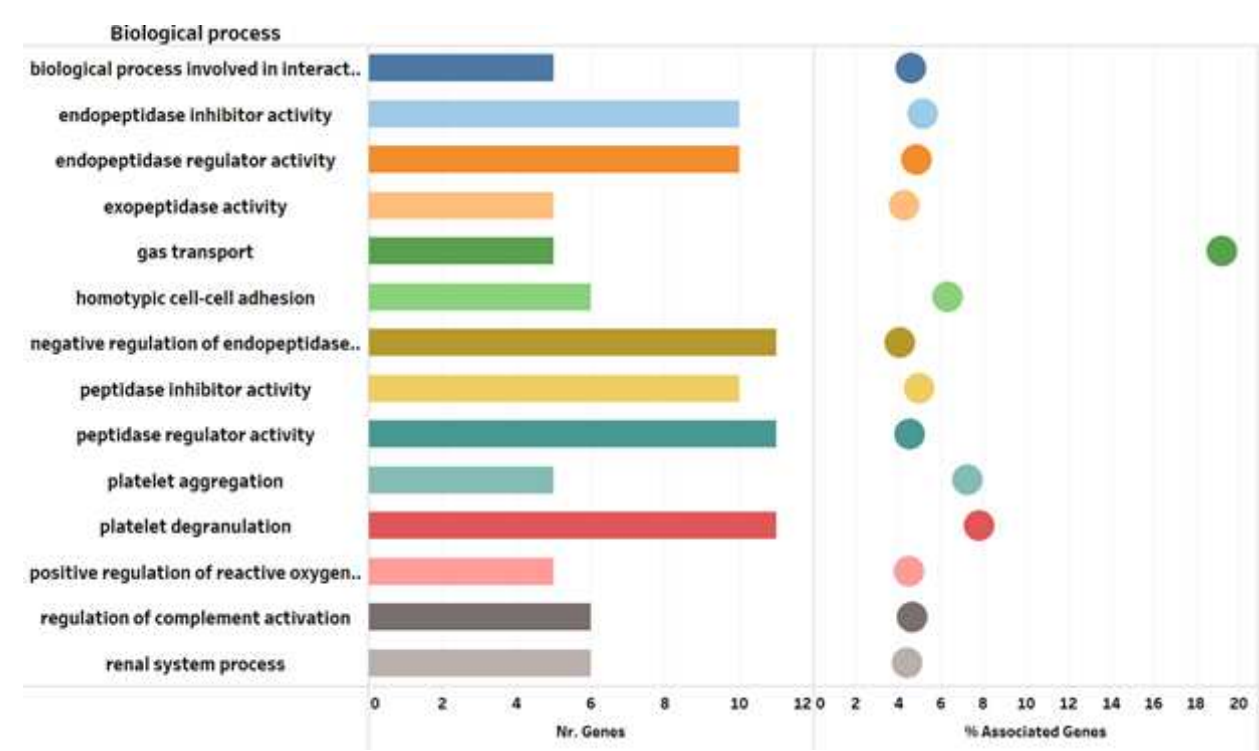

Figure 4: GO annotation of genes involved in biological process

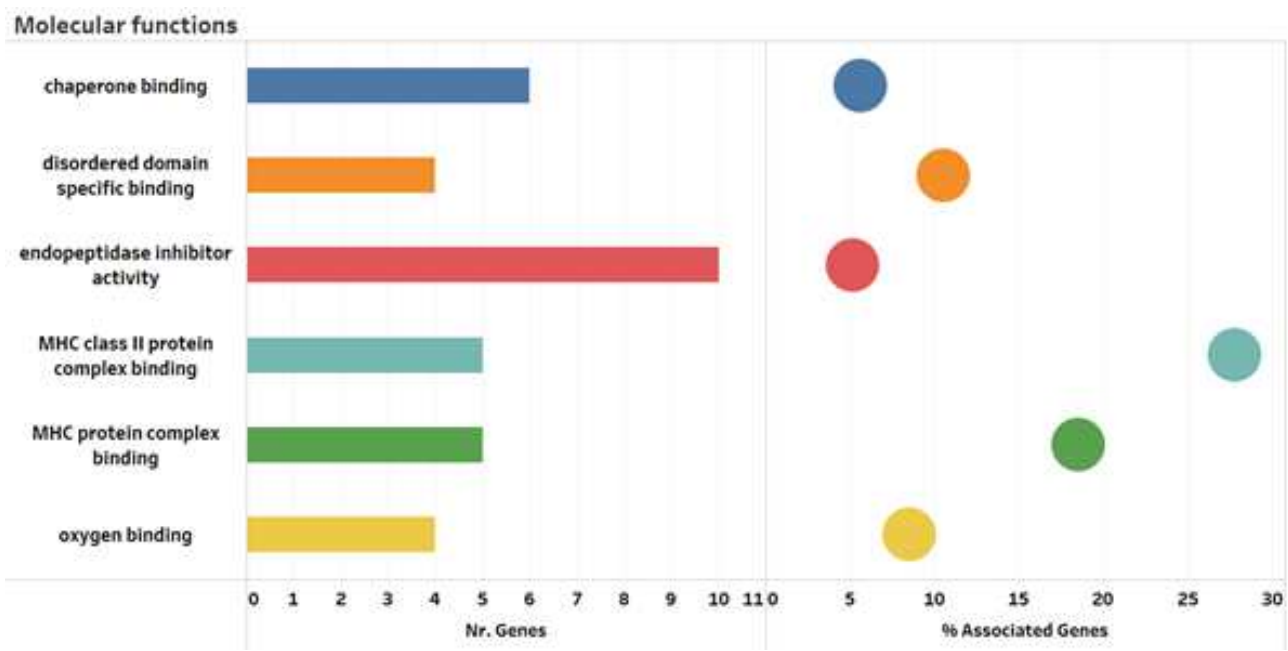

Figure 5: GO annotation of genes involved in molecular function 


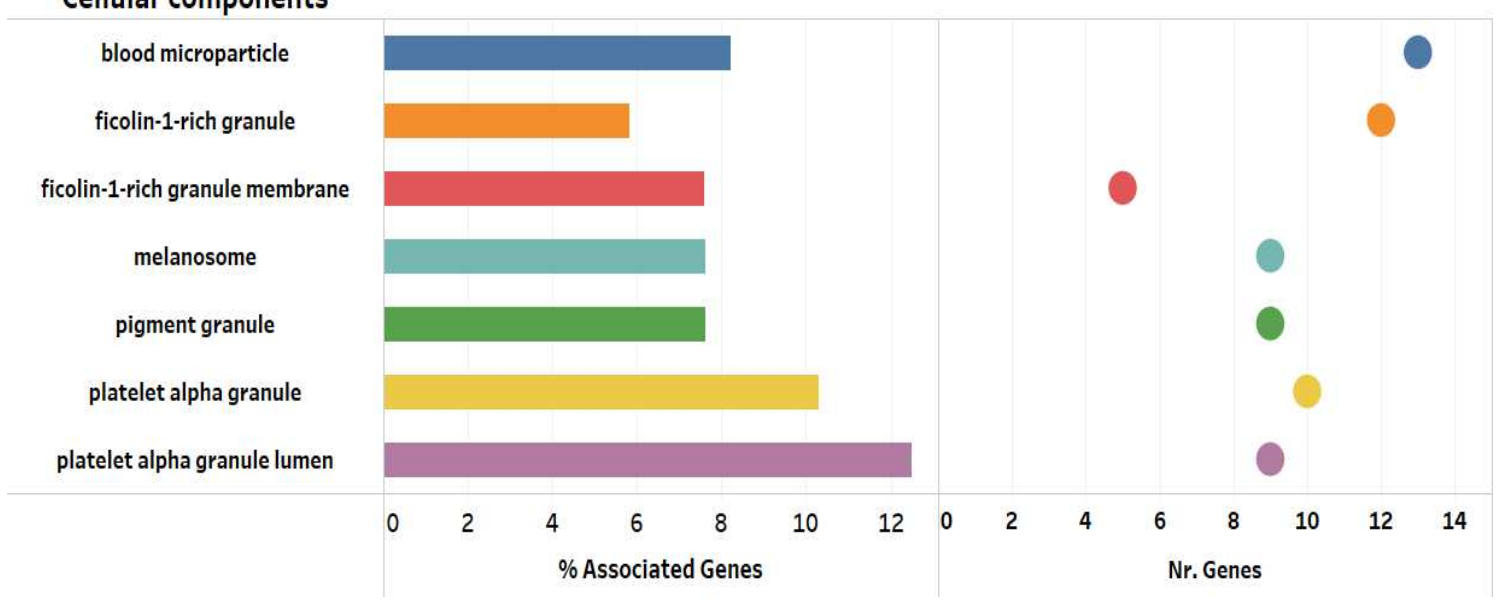

Figure 6: GO annotation of genes involved in cellular components

From the gene-disease associations, a final list of 8 genes were shortlisted and the hub network on the gene interaction is represented in Figure 7.

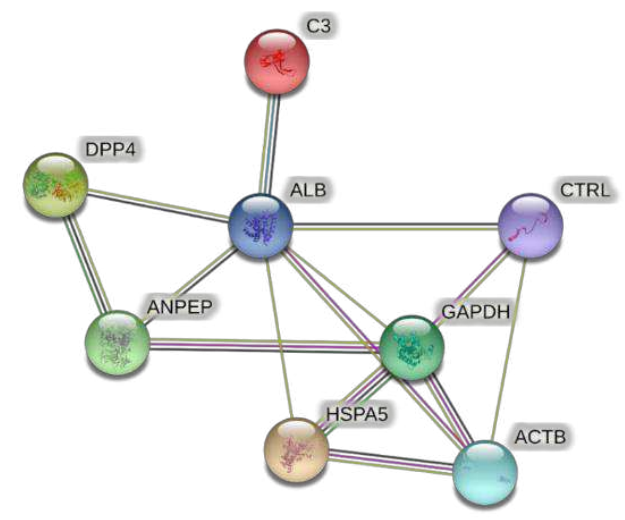

Figure 7: Hub gene interaction of the exosome biomarkers

\section{Discussion:}

Obesity is becoming a raising concern, especially in developing countries $(19,20)$. The increased energy intake and reduced energy breakdown causes release of endotoxins, increase in intestinal permeability results in low-grade chronic inflammation $(21,22)$. Integrative management strategies have been implemented to provide effective management lately(23). Due to the limitations in the present anthropometric measures, determination of specific markers is a necessity(24-26).

In this study, we attempted to identify biomarkers of Obesity from participants who underwent Ayurveda treatment (Lekhana Basti) a method of using medicated enema for restoring gut integrity with the purpose of reducing obesity $(27,28)$. We used exosomes from urine as the 
source of biomarkers. While, many studies have reported adipose-tissue derived exosome markers in obesity (29-31), in this study, for the first time, we use urine (a non-invasive source of sample) to isolate exosomes from obese individual to identify biomarkers of disease. From the LC-MS/MS analysis, we identified exosome specific proteins (CD3, CD81, CD63), which validates the isolation and purification method used in the study(32).

Obesity is seen with dysregulation of glucose, protein, vitamin and immune pathways such as upregulation of cell adhesion, transmembrane migration activities, and increased amino acid, protein and glucose regulation pathways, upregulation in circulatory immune and inflammatory process, enhanced matrix part, protein, peptidase, extracellular matrix bindings (33-35). In our study, KEGG pathway enrichment analysis showed 12 enriched pathways (73 genes) related to metabolic dysregulations where, 19 genes were mapped to the dysregulated metabolisms, 7 genes were responsible for protein digestion and regulation, followed by upregulation of genes associated with glycolysis and gluconeogenesis, pancreatic secretion, and gastric acid secretion. From the over-representation analysis, 31 molecular pathways were overexpressed at a minimum of >11 times fold enrichment. Interestingly, the top fold enrichment was observed in metallopeptidase(MMP) activity at $>100$ fold change. MMPs are proteinases associated with matrix degradation, adipogenesis and differentiation in obesity and cardiovascular conditions(36,37). Our results indicate the significant rise in MMP activities directly correlates with obesity. Most of the overrepresented pathways from our results were associated to intestinal transmembrane activity including dipeptidyl-peptidase activity, sugar transmembrane transporter, hexose, glucose, carbohydrate transmembrane activities as seen in $\operatorname{obesity}(38-41)$.

From the functional annotation analysis, the peptidase regular and inhibitor activity were overexpressed in biological processes, ficolin-1-granule was high in cellular functions, and negative regulation of peptidase activity, peptidase inhibitor activity, and endopeptidase activities were the major molecular functions significantly affected, as indicated in literature. Interestingly, many of the common genes associated with obesity were mapped to cancer, and metabolic diseases as reported in many studies (41-46).

Finally, using urine exosomes as source of biomarkers, we found 8 hub genes related to obesity (HSPA5, C3, GAPDH, ACTB, ALB, DPP4, ANPEP and CTRL) related to obesity. We found that the 8 biomarkers showed a differential expression pattern that is similar to signatures of 
obesity. Studies show that an increased interaction of Reticulon-3 with Heat-Shock Protein 5 (HSPA5) activates SREBP-1 which induces obesity and accumulation of triglycerides(47). Increased Serum Complement(C3) shows significant relation to adipose tissue variations in obese conditions $(48,49)$. GAPDH, one of the housekeeping gene which plays a major role in glucose and nuclear functions, is overexpressed under obese conditions, affecting major metabolic pathways(50-53). Similarly increased expression of Serum Albumin(ALB), an indicator leading to morbid obesity(54), and under-expression of Actin(ACTB), is seen in obese conditions(52). DPP4, an adipokine made in the adipose tissues is positively related to obesity(39). Similarly, ANPEP, which encodes metalloprotease, an highly glycosylatedprotein which increases with increased adipogenesis in obesity(55). Finally, Chymotrypsin(CTRL), is a protease inhibitor required for insulin metabolism is downregulated in obesity(56). Our biomarker data shows a 'correction pattern' of these biomarkers from the pre-post treatment analysis. Biomarkers HSPA5, C3, GAPDH, ALB, DPP4 and ANPEP, which are overexpressed in obesity, were reduced post-treatment. Further, CTRL and ACTB which are under expressed under obese conditions, had improved expression levels post treatment. This study is the first exosome biomarker study to report on urine exosome biomarkers change in obesity post Ayurveda treatment.

\section{Conclusion}

Due to the complex pathophysiology of obesity, the diagnosis and treatment has become challenging. To overcome the diagnostic limitations of the current anthropometric measures, this study has used urine exosomes as markers of obesity treatment. The study outcomes, pathway enrichment and functional annotation indicated dysregulated metabolic pathways and functions closely associated with obesity. The abundance of dysregulation of 8 hub genes which represent urine exosome biomarkers related to obesity was reduced in the post-treatment biomarker group indicating the potential of Ayurveda treatment (Lekhana Basti) in correcting the metabolic dysfunctions, altering the intestinal permeability and gut regulation. This is the first study to have identified urine exosome biomarkers of obesity and its differential expression changes after Ayurvedic treatment in Indian female subjects. However, this study is not void of limitations, a larger study sample size may re-validate the specific urine exosome biomarker of treatment. An investigation of holistic treatment methods into underlying mechanisms is still pending. Future studies can help identify clinical diagnostic exosome markers and mechanism of action of holistic treatments such as Ayurveda. However, this study 
supports the need and acceptance of integrative health approaches to effectively overcome obesity.

\section{Data Availability}

The dataset supporting the findings of this paper belongs to the TDU, Bangalore, India. Currently, the data are not publicly available but can be obtained upon reasonable request and with permission from the corresponding author, Bhavya Vijay (bhavya.v@tdu.edu.in).

\section{Conflict of Interest}

The authors declare that there are no conflicts of interest regarding this paper.

\section{Acknowledgements}

We thank Prof. Darshan Shankar, Vice Chancellor, TDU for his support, encouragement and for providing a platform and resources in conducting the research. We also thank our colleagues and friends who supported us throughout the work.

\section{Supplementary Materials}

See Supplementary excel file for details on final expression profile of the urine exosome proteins.

\section{References}

1. Luhar S, Timæus IM, Jones R, Cunningham S, Patel SA, Kinra S, et al. Forecasting the prevalence of overweight and obesity in India to 2040. PLoS ONE. 2020.

2. Girdhar S, Sharma S, Chaudhary A, Bansal P, Satija M. An epidemiological study of overweight and obesity among women in an Urban area of North India. Indian J Community Med. 2016;

3. Barnes AS. The epidemic of obesity and diabetes. Cardiovasc Dis women. 2011;

4. Jiang SZ, Lu W, Zong XF, Ruan HY, Liu Y. Obesity and hypertension. Experimental and Therapeutic Medicine. 2016.

5. Landsberg L, Aronne LJ, Beilin LJ, Burke V, Igel LI, Lloyd-Jones D, et al. ObesityRelated Hypertension: Pathogenesis, Cardiovascular Risk, and Treatment. J Clin Hypertens. 2013;

6. Hill JJ. Obesity: An Emerging Threat. J Natl Black Nurses Assoc. 2018;

7. Buchholz AC, Bugaresti JM. A review of body mass index and waist circumference as markers of obesity and coronary heart disease risk in persons with chronic spinal cord injury. Spinal Cord. 2005. 
8. Han TS, Sattar N, Lean M. ABC of obesity: Assessment of obesity and its clinical implications. British Medical Journal. 2006.

9. Wyatt HR. Update on treatment strategies for obesity. J Clin Endocrinol Metab [Internet]. 2013 Apr [cited 2020 Sep 1];98(4):1299-306. Available from: /pmc/articles/PMC3615205/?report=abstract

10. Katzmarzyk PT, Bray GA, Greenway FL, Johnson WD, Newton RL, Ravussin E, et al. Ethnic-Specific BMI and waist circumference thresholds. Obesity. 2011;

11. Iacomino G, Siani A. Role of microRNAs in obesity and obesity-related diseases. Genes and Nutrition. 2017.

12. Cui X, You L, Zhu L, Wang X, Zhou Y, Li Y, et al. Change in circulating microRNA profile of obese children indicates future risk of adult diabetes. Metabolism. 2018;

13. Oses M, Sanchez JM, Portillo MP, Aguilera CM, Labayen I. Circulating miRNAs as biomarkers of obesity and obesity-associated comorbidities in children and adolescents: A systematic review. Nutrients. 2019;

14. Lei LM, Lin X, Xu F, Shan SK, Guo B, Li FXZ, et al. Exosomes and Obesity-Related Insulin Resistance. Frontiers in Cell and Developmental Biology. 2021.

15. Zhou Y, Tan C. miRNAs in Adipocyte-Derived Extracellular Vesicles: Multiple Roles in Development of Obesity-Associated Disease. Frontiers in Molecular Biosciences. 2020 .

16. Obermaier C, Griebel A, Westermeier R. Principles of Protein Labeling Techniques. Vol. 2261, Methods in Molecular Biology. 2021. 549-562 p.

17. Rider MA, Hurwitz SN, Meckes DG. ExtraPEG: A polyethylene glycol-based method for enrichment of extracellular vesicles. Sci Rep. 2016;

18. Shevchenko A, Tomas H, Havliš J, Olsen J V., Mann M. In-gel digestion for mass spectrometric characterization of proteins and proteomes. Nat Protoc. 2007;1(6):285660.

19. Nour NN. Obesity in resource-poor nations. Rev Obstet Gynecol. 2010;

20. Bhurosy T, Jeewon R. Overweight and obesity epidemic in developing countries: A problem with diet, physical activity, or socioeconomic status? Scientific World Journal. 2014.

21. Sun L, Ma L, Ma Y, Zhang F, Zhao C, Nie Y. Insights into the role of gut microbiota in obesity: pathogenesis, mechanisms, and therapeutic perspectives. Protein and Cell. 2018.

22. Ott B, Skurk T, Hastreiter L, Lagkouvardos I, Fischer S, Büttner J, et al. Effect of 
caloric restriction on gut permeability, inflammation markers, and fecal microbiota in obese women. Sci Rep. 2017;

23. Heber D. An integrative view of obesity. In: American Journal of Clinical Nutrition. 2010.

24. Sommer I, Teufer B, Szelag M, Nussbaumer-Streit B, Titscher V, Klerings I, et al. The performance of anthropometric tools to determine obesity: a systematic review and meta-analysis. Sci Rep. 2020;

25. Gurunathan U, Myles PS. Limitations of body mass index as an obesity measure of perioperative risk. British Journal of Anaesthesia. 2016.

26. Mayoral LPC, Andrade GM, Mayoral EPC, Huerta TH, Canseco SP, Rodal Canales FJ, et al. Obesity subtypes, related biomarkers \& heterogeneity. The Indian journal of medical research. 2020.

27. Auti S, Thakar A, Shukla V, Ravishankar B. Assessment of Lekhana Basti in the management of hyperlipidemia. AYU (An Int Q J Res Ayurveda). 2013;

28. Auti SS, Ashok BK, Thakar AB, Shukla VJ, Ravishankar B. An experimental study to evaluate the pharmacokinetic aspect of Lekhana Basti (Emaciating/ Desiccating Medicated Enema). Anc Sci Life. 2011;

29. Ferrante SC, Nadler EP, Pillai DK, Hubal MJ, Wang Z, Wang JM, et al. Adipocytederived exosomal miRNAs: A novel mechanism for obesity-related disease. Pediatr Res. 2015;

30. Castaño C, Kalko S, Novials A, Párrizas M. Obesity-associated exosomal miRNAs modulate glucose and lipid metabolism in mice. Proc Natl Acad Sci U S A. 2018;

31. Zhao H, Shang Q, Pan Z, Bai Y, Li Z, Zhang H, et al. Exosomes from adipose-derived stem cells attenuate adipose inflammation and obesity through polarizing M2 macrophages and beiging in white adipose tissue. Diabetes. 2018;

32. Andreu Z, Yáñez-Mó M. Tetraspanins in extracellular vesicle formation and function. Front Immunol. 2014;

33. Li L, Wang G, Li N, Yu H, Si J, Wang J. Identification of key genes and pathways associated with obesity in children. Exp Ther Med. 2017;

34. Zhang Y, Zheng Y, Fu Y, Wang C. Identification of biomarkers, pathways and potential therapeutic agents for white adipocyte insulin resistance using bioinformatics analysis. Adipocyte. 2019;

35. Hsu YHH, Astley CM, Cole JB, Vedantam S, Mercader JM, Metspalu A, et al. Integrating untargeted metabolomics, genetically informed causal inference, and 
pathway enrichment to define the obesity metabolome. Int J Obes. 2020;

36. Chavey C, Mari B, Monthouel MN, Bonnafous S, Anglard P, Van Obberghen E, et al. Matrix metalloproteinases are differentially expressed in adipose tissue during obesity and modulate adipocyte differentiation. J Biol Chem. 2003;

37. Berg G, Schreier L, Miksztowicz V. Circulating and adipose tissue matrix metalloproteinases in cardiometabolic risk environments: Pathophysiological aspects. Hormone Molecular Biology and Clinical Investigation. 2014.

38. Pendergrass M, Bertoldo A, Bonadonna R, Nucci G, Mandarino L, Cobelli C, et al. Muscle glucose transport and phosphorylation in type 2 diabetic, obese nondiabetic, and genetically predisposed individuals. Am J Physiol - Endocrinol Metab. 2007;

39. Sarkar J, Nargis T, Tantia O, Ghosh S, Chakrabarti P. Increased Plasma Dipeptidyl Peptidase-4 (DPP4) Activity Is an Obesity-Independent Parameter for Glycemic Deregulation in Type 2 Diabetes Patients. Front Endocrinol (Lausanne). 2019;

40. Chadt A, Al-Hasani H. Glucose transporters in adipose tissue, liver, and skeletal muscle in metabolic health and disease. Pflugers Archiv European Journal of Physiology. 2020.

41. Poloz Y, Stambolic V. Obesity and cancer, a case for insulin signaling. Cell death \& disease. 2015.

42. Locke AE, Kahali B, Berndt SI, Justice AE, Pers TH, Day FR, et al. Genetic studies of body mass index yield new insights for obesity biology. Nature. 2015;

43. Lan N, Lu Y, Zhang Y, Pu S, Xi H, Nie X, et al. FTO - A Common Genetic Basis for Obesity and Cancer. Frontiers in Genetics. 2020.

44. Kolb R, Sutterwala FS, Zhang W. Obesity and cancer: inflammation bridges the two. Current Opinion in Pharmacology. 2016.

45. Tsilidis KK, Helzlsouer KJ, Smith MW, Grinberg V, Hoffman-Bolton J, Clipp SL, et al. Association of common polymorphisms in IL10, and in other genes related to inflammatory response and obesity with colorectal cancer. Cancer Causes Control. 2009;

46. Cleveland RJ, Gammon MD, Long CM, Gaudet MM, Eng SM, Teitelbaum SL, et al. Common genetic variations in the LEP and LEPR genes, obesity and breast cancer incidence and survival. Breast Cancer Res Treat. 2010;

47. Xiang R, Fan L-L, Huang H, Chen Y-Q, He W, Guo S, et al. Increased RTN3 Leads to Obesity and Hypertriglyceridemia by Interacting with HSPA5. Circulation. 2018;

48. Karkhaneh M, Qorbani M, Mohajeri-Tehrani MR, Hoseini S. Association of serum 
complement C3 with metabolic syndrome components in normal weight obese women. J Diabetes Metab Disord. 2017;

49. Shim K, Begum R, Yang C, Wang H. Complement activation in obesity, insulin resistance, and type 2 diabetes mellitus. World J Diabetes. 2020;

50. Dugail I, Quignard-Boulange A, Bazin R, Le Liepvre X, Lavau M. Adipose-tissuespecific increase in glyceraldehyde-3-phosphate dehydrogenase activity and mRNA amounts in suckling pre-obese Zucker rats. Effect of weaning. Biochem J. 1988;

51. Rolland V, Dugail I, Le Liepvre X, Lavau M. Evidence of increased glyceraldehyde-3phosphate dehydrogenase and fatty acid synthetase promoter activities in transiently transfected adipocytes from genetically obese rats. J Biol Chem. 1995;

52. Fan X, Yao H, Liu X, Shi Q, Lv L, Li P, et al. High-Fat Diet Alters the Expression of Reference Genes in Male Mice. Front Nutr. 2020;

53. Moraes RC, Blondet A, Birkenkamp-Demtroeder K, Tirard J, Orntoft TF, Gertler A, et al. Study of the Alteration of Gene Expression in Adipose Tissue of Diet-Induced Obese Mice by Microarray and Reverse Transcription-Polymerase Chain Reaction Analyses. Endocrinology. 2003;

54. Mosli RH, Mosli HH. Obesity and morbid obesity associated with higher odds of hypoalbuminemia in adults without liver disease or renal failure. Diabetes, Metab Syndr Obes Targets Ther. 2017;

55. Iaffaldano L, Nardelli C, Raia M, Mariotti E, Ferrigno M, Quaglia F, et al. High aminopeptidase N/CD13 levels characterize human amniotic mesenchymal stem cells and drive their increased adipogenic potential in obese women. Stem Cells Dev. 2013;

56. Hamel FG, Upward JL, Bennett RG. In vitro inhibition of insulin-degrading enzyme by long-chain fatty acids and their coenzyme A thioesters. In: Endocrinology. 2003. 


\section{Supplementary Files}

This is a list of supplementary files associated with this preprint. Click to download.

- supplementaryfilepreprint.xlsx 